\title{
SCHISTOSOMA MANSONI: NÍVEIS DE INFECCIOSIDADE PARA OS MOLUSCOS HOSPEDEIROS INTERMEDIÁRIOS DO BRASIL
}

\author{
Cecilia Pereira de Souza, Liana K. Jannotti-Passos, Iaci Belo de F. \\ Vieira e Ivan Barbosa Machado Sampaio
}

\begin{abstract}
Os nineis de infecciosidade de Schistosoma mansoni para as três espécies de Biomphalaria, bospedeiras intermediárias do parasita no Brasil, foram pesquisados após exposiçóes conjuntas dos moluscos a miracidios, no laboratório e no campo. Foram utilizadas as cepas LE e SJ de S. mansoni, mantidas no laboratório e WVS e RFS obtidas de fezes de escolares de Belo Horizonte, $M G$. Os resultados mostraram a infecciosidade maior de $\mathrm{S}$. mansoni para $\mathrm{B}$. glabrata, com taxas de infecção de 4,7 a $85,5 \%$. A B. straminea foi suscetinel as cepas $L E$. SJ e WVS, com taxas de infeccăo de 11, 0 a $24,6 \%$. A B. tenagophila foi suscetivel a LE e SI com taxas de infeccão de 2,5 a 6,5\% As médias de cercárias da cepa WVS, eliminadas por dia por B. straminea e B. glabrata variaram de $93 \pm 59$ e $782 \pm 1.120$, respectivamente.
\end{abstract} Brasil.

Palauras-chates: Schistosoma mansoni. Infecciosidade. Moluscos bospedeiros

A larga distribuição geográfica dos moluscos hospedeiros de Schistosoma mansoni no Brasil, onde a B. glabrata é encontrada em 17 dentre 27 unidades federativas, a $B$. straminea em 23 e a $B$. tenagophila em $10^{13} 1+1516293131$, nos levou ao estudo do nível de infecciosidade do trematódeo para as três espécies de moluscos. A importância epiclemiológica de $B$. glabrata nas regiòes onde ela ocorre é indiscutivel. $A B$. straminea é um vetor importante na regiâo nordeste e a $B$. tenagophila nas regiões sudeste e sul ${ }^{223+678121825}$.

Em Minas Gerais levantamentos malacológicos diversos já registraram a ocorrência de $B$. glabrata em 167 municípios, de $B$. straminea em 74 e de B. tenagophila em $31^{5} 1315$ 28. Em alguns municípios mineiros ocorrem duas ou as três espécies hospedeiras, como em Belo Horizonte, Jaboticatubas, Coronel Fabriciano, Governador Valadares, Frei Inocêncio, Engenheiro Caldas, Nova Era, Dom Joaquim, dentre outras 131528 . A

Centro de Pesquisas "René Rachou"- Fundação Oswaldo Cruz e Escola de Veterinária, Universidade Federal de Minas Gerais, Belo Horizonte, $M G$.

Apoio financeiro: $\mathrm{CNPq}$.

Endereço para correspondência: Dra Cecília Percira de Souza. Laboratório de Malacologia - Centro de Pesquisas "René Rachou"/FIOCRUZ. Caixa Postal 1743, 30161-970 Belo Horizonte, MG. Fax: 031.295.3115.

Recebido para publicação $\mathrm{em} \mathrm{27/03/95.}$ ocorrência de duas ou três espécies em uma mesma região, possibilita o encontro eventual dessas espécies juntas em um mesmo criadouro.

No presente estudo, foram feitas infecções experimentais conjuntas das três espécies de Biomphalaria, com diferentes cepas de $S$. mansoni, no laboratório e no campo, com o objetivo de conhecer melhor o comportamento do trematódeo e dos moluscos hospedeiros em criadouros onde eventualmente podem ser encontradas duas ou três espécies, como ocorre na região da Pampulha, em Belo Horizonte ${ }^{30}$.

\section{MATERIAL E MÉTODOS}

Foram usados moluscos Biomphalaria criados no laboratório ou capturados no campo: a) B. glabrata, criada no laboratório, originária de exemplares coletados no Barreiro de Cima, Belo Horizonte, (MG) e mantida no laboratório há mais de 20 anos; foram usados moluscos medindo $4-10 \mathrm{~mm}$ de diâmetro. b) $B$. tenagophila, descendente de exemplares procedentes de Campinas (SP) em 1991 e outra população capturada no lago da Pampulha, Belo Horizonte em 1988; os moluscos usados mediam 4-10 mm. c) $B$. straminea, descendente de exemplares capturados em vala localizada em sítio nas margens do lago da Pampulha, Belo Horizonte, em 1988 e 1989. Parte dos 
Souza CP, Jannotti-Passos LK, Vieira IBF, Sampato IBM. Schistosoma mansoni: nineis de infecciosidade para os moluscos hospedeiros intermediários do Brasil. Revista da Sociedade Brasileira de Medicina Tropical 29:1116, janl-fet, 1996.

moluscos coletados em 1988 foi usada em experimento no campo; foram utilizados exemplares com $4-7 \mathrm{~mm}$.

Foram usadas quatro cepas de S. mansoni: duas mantidas no laboratório; LE, de Belo Horizonte (MG) e SJ de São José dos Campos (SP). As outras duas foram obtidas de fezes de escolares de Belo Horizonte; um residente na Pampulha, WVS, isolada em 1988, e outro da região de Gorduras, RFS, isolada em 1991.

\section{Infecçào no laboratório e no campo.}

a) No láboratório os ovos de S. mansoni foram obtidos de fígado de hamster, após 48 a 50 clias de infeccáo, segundo técnica de Pellegrino e Katz com modificações ${ }^{20}$. Duzentos moluscos de cada espécie foram expostos em conjunto a cerca de 50 miracidios por exemplar. Cinqüenta exemplares de cada espécie foram colocados dentro de pequenas gaiolas de forma cilíndrica, de tela de metal $(11,0 \times 3,5 \mathrm{~cm})$, con tampa de plástico. Seis gaiolas contendo 300 moluscos foram submersas em $7000 \mathrm{ml}$ de água contendo cerca de 15.000 miracídios. dentro de um cristalizador com tampa. O tempo de exposiçáo foi de 6 horas, sob luz artificial, em temperatura de $27,0 \pm 1{ }^{\circ} \mathrm{C}$.

b) No campo, foram usados ovos de $S$. mansoni obtidos de fezes coletadas no dia do experimento. O material foi pesado e retirouse uma amostra para determinar o número médio de ovos por grama de fezes". No primeiro experimento foram usadas $140 \mathrm{~g}$ de fezes do paciente WVS, contendo cerca de 601.440 ovos de $S$. mansoni; no segundo, foram usadas $42 \mathrm{~g}$ de fezes do paciente RFS, contendo cerca de 42.720 ovos. As fezes foram previamente suspensas em bequer contendo água dos poços onde posteriormente foram lançadas. Cento e cinquienta moluscos de cada espécie foram colocados em três gaiolas com tampa; 9 gaiolas contendo 450 moluscos foram fixadas em três estacas de madeira e submersas na água de um mesmo poço. A suspensão de fezes foi lançada sobre os locais onde estavam os moluscos. O tempo de exposição foi de 6 horas em temperatura de $25,0^{\circ} \mathrm{C}$ no primeiro experimento e de 23,5 a $24,0^{\circ} \mathrm{C}$ no segundo. $\mathrm{O}$ pH da água variou de 5 a 6 durante as exposiçôes. Foram utilizados poços pequenos com cerca de 1501 de água ${ }^{22}$, pertencentes a sistema de valas de irrigação de horta, localizado no Barreiro de Baixo, Belo Horizonte, criadouro de B. glabrata. No dia do experimento a entrada e saída da água dos poços foram fechadas para evitar o carreamento do material em suspensão. Após a exposição, as gaiolas contendo os moluscos foram recolhidas e levadas para o laboratório. Os exemplares de cada espécie foram colocados em aquários separados e mantidos em condiçôes iguais, em temperatura de 21,0 a $28,0^{\circ} \mathrm{C}$. Após a retirada dos moluscos os poços foram tratados com $1000 \mathrm{ml}$ de soluçào de hipoclorito de sódio (água sanitária) para eliminar os miracídios e outros organismos provenientes das fezes.

Decorridos trinta dias da exposição os moluscos do laboratório foram examinados em estereomicroscópio após esmagamento entre lâminas de vidro para detectar a presença de esporocistos ou cercárias. Os moluscos expostos no campo foram examinados após exposição à luz e os exemplares positivos foram separados e colocados individualmente em aquários de plástico, com 400ml de água. No experimento com a linhagem wVS os exemplares negativos foram examinados até 70 dias e depois esmagados. No expermento $\mathrm{com}$ RFS os moluscos negativos foram examinados após exposição à luz aos 30 dias e após esmagamento aos 37 dias após a exposiçào.

Os moluscos infectados com a linhagem WVS tiveram suas cercárias contadas em 4 alíquotas de $1 \mathrm{ml}$, retiradas dos aquários 3 vezes por semana, antes da troca de água. A data da morte de cada molusco infectado foi registrada.

Procedeu-se a avaliação da compatibilidade dos moluscos das três espécies à infecçào com a linhagem WVS, utilizando o método de Frandsen". Para o cálculo do índice TCP/100, tomoulse o total de cercárias eliminadas durante a vida dos moluscos, multiplicou-se pelo número de exemplares expostos e dividiu-se por 100 . Os índices obtidos foram comparados com dados da tabela de Frandsen" que contém 6 classes de compatibilidade, segundo o número de cercárias eliminadas, além da classe 0 de resistente.

Análise estatística - as diferenças numéricas obtidas nos experimentos foram avaliadas $\mathrm{com}$ aplicação do teste do qui-quadrado. As 
Souza CP, Jannotti-Passos LK, Vieira IBF, Sampaio IBM. Schistosoma mansoni: níteis de infecciosidade para os moluscos bospedeiros intermediários do Brasil. Revista da Sociedade Brasileira de Medicina Tropical 29:1116, jan-fev, 1996.

variáveis quantitativas foram submeticlas a análise de variância com as médias comparadas pelo teste $t$ de Student. A variável número médio de cercárias $(\mathrm{x})$ sofreu transformação logarítmica $\log (\mathrm{x}+1)$ por apresentar desvios proporcionais às médias ${ }^{2+}$. Em todos os cálculos foi adotado o nível de significância de $5 \%(\mathrm{p}</ 0,05)$.

\section{RESULTADOS}

Os resultados das exposições conjuntas dos moluscos das três espécies a diferentes linhagens de $S$. mansoni no laboratório e no campo são mostrados na Tabela 1. As diferenças entre as taxas de infecção conjuntas foram estatisticamente significativas $(p<0,05)$. Os níveis maiores de infecciosidade dos miracídios das linhagens LE, SJ, WVS e RFS foram para B. glabrata. Com a linhagem LE, as diferenças foram estatisticamente significativas entre $B$. tenagophila e $B$. straminea $\mathrm{x}^{2}=12,42$; entre B. glabrata e B. tenagopbila, $\mathrm{x}^{2}=291,9$; entre $B$. glabrata e B. straminea, $\mathrm{x}^{2}=242,47$; entre $B$. glabrata e $B$. straminea, $\mathrm{x}^{2}=213,68 \mathrm{e}$ não significativa entre $B$. straminea $\mathrm{e} B$. tenagopbila (Tabela 1). A preferência maior dos miracídios da linhagem WVS foi para $B$. straminea e $B$. glabrata, diferença não significativa estatisticamente; entre $B$. straminea e $B$. tenagophila a diferença foi estatisticamente significativa, $\mathrm{x}^{2}=137,05$ assim como entre B. glabrata e B. tenagophila, $\mathrm{x}^{2}=$ 32,23. A B. tenagophila não se infectou com WVS.

Com a linhagem RFS as diferenças entre as taxas de infecção foram estatisticamente significativas. A infecciosidade dos miracídios da RFS foi maior para $B$. glabrata, $\mathrm{x}^{2}=87,21$, em relação à $B$. straminea e $B$. tenagophila que não se infectaram (Tabela 1).
As médias de cercárias da linhagem WVS, eliminadas por molusco, por dia foram de $93 \pm$ 59 para $B$. straminea e de $782 \pm 1.120$ para $B$. glabrata, significativamente maior para a segunda espécie (teste $t$ de Student).

O cálculo do índice TCP $/ 100$ possibilitou a classificação da compatibilidade de cada espécie com a linhagem WVS. Assim para $B$. glabrata foi de $1.990 .959 \times 100 / 150=$ 1.327.063, classe VI, extremamente compativel

Os índices obtidos para cada espécie são apresentados na Tabela 2 .

As medianas de mortalidade dos moluscos infectados com WVS mostraram que $50 \%$ de $B$. straminea morreram até 71 dias e de $B$. glabrata até 92 dias após a infeç̧ào. A taxa de sobrevivência dos moluscos infectados, após 90 dias foi de $18,9 \%$ para $B$. straminea e de $65,5 \%$ para B. glabrata. A longevidade maior foi de 16 semanas ( 112 dias) para $B$. straminea e de 33 semanas (231 dias) para $B$ glabrata, de exemplar com infecção bissexual .

\section{DISCUSSÃO}

Os resultados obtidos no laboratório com as linhagens LE e SJ mostraram através das taxas de infeccão conjunta os niveis maiores de infecciosidade de $S$ mansoni das duas linhagens para $B$. glabrata, seguindo-se a $B$. straminea e foi menor para a B. tenagopbila. (Tabela 1). Na infecção com a linhagem SJ mais adaptada à $B$. tenagopbila ${ }^{17}$ 18 19 , a infecciosidade maior do trematódeo foi para $B$. glabrata e semelhante para $B$. tenagophila e $B$. straminea (Tabela 1). A linhagem SJ, tem sido mantida em B. glabrata, o que justifica esses resultados, mas em infeccôes individuais normalmente a $B$. tenagophila apresenta taxa de infecção significativamente maior do que a de B. straminea com essa cepa ${ }^{27}$.

Tabela 1 - Nitueis de infecciosidade de diferentes linhagens de Schistosoma mansoni para Biomphalaria em exylesiçies conjuntas.

\begin{tabular}{|c|c|c|c|c|}
\hline \multicolumn{2}{|c|}{ Molusco } & \multirow{2}{*}{$\begin{array}{l}\text { Linhagem de } \\
\text { S. mansoni }\end{array}$} & \multirow{2}{*}{$\begin{array}{l}\text { Local de } \\
\text { exposição }\end{array}$} & \multirow{2}{*}{$\begin{array}{l}\text { Tuxis de } \\
\text { infecciol }\end{array}$} \\
\hline espécie & procedência & & & \\
\hline \multirow[t]{4}{*}{ B. tenlagophila } & Campinas-SP & LE & laboratorio & 2.5 \\
\hline & Campinas-SP & $\mathrm{SI}$ & laboratorio & 6.5 \\
\hline & Pampulha-MG* & WVS & campo & 0.0 \\
\hline & Pampulha-MG & RFS & catmpo & 0.0 \\
\hline \multirow[t]{4}{*}{ B. straminea } & Pampulha-MG & LE & laboratório & 11,5 \\
\hline & Pampulha-MG & s! & laboratório & 11,0 \\
\hline & Pampulha-MG* & WVS & campo & 24,6 \\
\hline & Pampulha-MG & RFS & campo & 0,0 \\
\hline \multirow[t]{4}{*}{ B. glabrata } & Laboratório-MG & LE & laboratórios & 85.5 \\
\hline & Laboratório-MG & $\mathrm{sJ}$ & laboratório & 84,0 \\
\hline & Laboratório-MG & WVs & cumpo & 19.3 \\
\hline & Laboratório-MG & RFS & campo & 4.7 \\
\hline
\end{tabular}

- Moluscos capturados no campo 
Souza CP, Jannotti-Passos LK, Vieira IBF, Sampaio IBM. Schistosoma mansoni: niveis de infecciosidade para os moluscos hospedeiros intermediärios do Brasil. Revista da Sociedade Brasileira de Medicina Tropical 29: 11 16, jan-fev, 1996.

Nas infecçoes no campo, os miracídios da linhagem WVS se desenvolveram somente em B. straminea e B. glabrata. Os níveis de infecciosidade maiores foram para B.straminea $(24,6 \%)$ e $B$. glabrata $(19,3 \%)$ e nula para $B$. tenagophila $(0.0 \%)$. No criadouro de $B$. straminea eventualmente é encontrada $B$. glabrata e ambas se infectaram experimentalmente com $S$. mansoni de cepa local em níveis semelhantes. Como não foram realizados cortes histológicos em $B$. tenagophila exposta a miracídios, não sabemos se os miracídios não penetraram ou se alguns penetraram e foram destruídos pelo sistema interno de defesa do hospedeiro. A $B$. tenagophila da Pampulha, Belo Horizonte, é suscetivel experimentalmente as linhagens LE e SI de S. mansoni, apresentando taxas de infecalo de 24,0 e $38.6 \%$, respectivamente e às cepas locais do trematódeo, apresentando laxa de infecciò natural de $0,03 \%$ '.

Os miracídios da linhagem RFS mostraram nível de infecciosidacle maior para $B$. glabrata, pois, as outras duas espécies nào eliminaram cercárias, provavelmente devido ao número menor de miracídios disponíveis e as condiçoes climáticas do dia, parcialmente nublado e com chuva fraca. $\Lambda$ temperatura da agua durante a exposição baixou de $24,0^{\circ} \mathrm{C}$ para $23,5^{\circ} \mathrm{C}$ no periodo experimental.

Os resultados obtidos em condiçòes seminaturais mostraram que na presença de $B$. glabrata e B. straminea a taxa de infecção de B. tenagophila da Pampulha foi nula com a linhagem WVS, procedente daquele bairro. De fato, em várias capturas de moluscos efetuadas durante vários anos naquele local e examinados nesse laboratório não foram encontradas $B$. tenagophila com infecção natural junto com B. glabrata. Por outro lado, a $B$. straminea da Pampulha, apesar de não ser capturada com infecção natural apresentou taxa de infecçào experimental semelhante à de B. glabrata, mostrando que ambas podem ser hospedeiras do trematódeo no mesmo habitat.
A média de cercárias eliminadas por $B$. straminea $(93 \pm 59)$ foi significativamente menor do que a de $B$. glabrata $(728 \pm 1.120)$ (teste $t$ de Student) devido provavelmente a destruição de parte dos esporocistos pelo sistema interno de defesa do hospedeiro. Entretanto a taxa de infecçào foi significativamente maior do que a dos moluscos de Paracatu ${ }^{27}$. O total de cercárias eliminadas por 37 exemplares de $B$. straminea, em 16 semanas, foi de 70.252 larvas, número 28 vezes menor do que o eliminado por $B$. glabrata (1.990.595) em 33 semanas, devido a longevidade maior e número mais elevado de cercárias eliminadas por essa espécie, o que está de acordo com as observações de Barbosa e Coelho ${ }^{2}$. Alguns exemplares de B. straminea interromperam a eliminação de cercárias por 1 a 5 dias mas nào houve cura parasitológica.

Os indices TCP/ 100 , obtidos para as três espécies de moluscos mostraram que a $B$. straminea foi pouco compativel com a linhagem WVS (classe II) e a B. tenagophila mostrou-se resistente ao trematódeo (classe 0) em presença das duas outras espécies (Tabela 2). Os resultados obtidos com B. straminea da Pampulha foram semelhantes aos dados registrados por Souza e cols em infeccaio individual de exemplares de Paracatu, MG com as linhagens LE e AL (classe II) $e$ inferiores aos obtidos por Paraense e Correa ${ }^{21}$, para molusco semelhante à $B$. straminea de Espinillar, Uruguai, infectado com SI, que foi compativel (classe III).

Apesar de menos suscetiveis e compatíveis do que a $B$. glabrata, e da infecciosidade igual ou menor do S. mansoni para essas duas espécies, devido a distribuição geográfica de ambas, aumenta o risco da expansão da esquistossomose em áreas indenes. De fato, Silva ${ }^{25}$ recentemente relatou o encontro de $B$. tenagophila com infecçào natural em Ouro Branco, MG, aumentando para quatro $O$ número de municípios mineiros onde o molusco é vetor.

Tabela 2 - compatibilidade de Biomphalana de Belo Horizonte, MG, com a linhagem Whs de Schistosoma mansoni, em exposicâ conjunta, nio campos.

\begin{tabular}{|c|c|c|c|c|}
\hline \multicolumn{2}{|c|}{ Molusco } & \multirow{2}{*}{$\begin{array}{l}\text { Indice } \\
\mathrm{TCP} / 100\end{array}$} & \multicolumn{2}{|c|}{ Compatibilidade } \\
\hline especic & procedénciát & & classe & descriçào \\
\hline B. tenagofbilat & Pampulha & 0 & 0 & incompativel \\
\hline B. stramines ${ }^{*}$ & Pampulha & 46.835 & U & pouco compativel \\
\hline B. glabrata & Laboratório & 1.327 .063 & VI & extremamente compativel \\
\hline
\end{tabular}

* Moluscos capturados no campo. 
Souza CP, Jannotti-Passos $L K$, Vieira IBF, Sampaio IBM. Schistosoma mansoni: niveis de infecciosidade para os moluscos bospedeiros intermediários do Brasil. Revista da Sociedade Brasileira de Medicina Tropical 29:1116, jan-fet, 1996.

Esses relatos, vem confirmar as observações de Paraense e Correa ${ }^{20}$, sobre 0 risco de expansâo da esquistossomose nas regiões sudeste e sul e o papel importante representado por $B$. tenagophila, como vetora, nessas regiões. Por outro lado, existe o risco da expansão da esquistossomose também na região amazônica onde a $B$. straminea ocorre em várias localidades ${ }^{1+31}$.

\section{SUMMARY}

The letels of infectinity of Schistosoma mansoni for the three species of Biomphalaria, intermediate bosts of parasite in Brazil were studied after exposing of molluscs to miracidia in the laboratory and in the field. The $L E$ and $S J$ strains of $S$. mansoni, maintained in laboratory were used in these experiments as well as the WVS and RFS strains obtained from faeces of schoolchildren from Belo Horizonte, MG. The results show the bigh letel of infectivity of $S$. mansoni for B. glabrata wilh infection rates varying from 4.7 to $85.5 \%$. The snatl B. straminea was susceptible to LE, SJ and WVS strains, with infection rates of 11.0 to $24.6 \%, B$. tenagophila was susceptible only to LE and SJ strains with infection rates of 2.5 to $6.5 \%$. The mean number of cercariae of the WVS strain shed per day, by $B$. straminea and B. glabrata were $93 \pm 59$ and $782 \pm 1,120$, respectively.

Key-words: Scbistosoma mansoni. Infectivity. Snail hosts. Brazil.

\section{AGRADECIMENTOS}

Ao Dr. Luiz Cândido de Souza Dias, da Universidade de Campinas, Sào Paulo, pelo fornecimento de amostra de moluscos de Campinas.

\section{REFERÊNCIAS BIBLIOGRÁFICAS.}

1. Alencar JE, Rouquayrol MZ, Figueiredo JS, Bezerra OFA. A esquistossomose no Ceará, análise do problema atual e sugestões para controle. Revista Brasileira de Malariologia e Doenças Tropicais 30:99-121, 1978.

2. Barbosa FS, Coelho MV. Qualidades de vetor dos hospedeiros de S. mansoni no nordeste do Brasil. I Susceptibilidade de A. glabratus e T. centimetralis à infecção por $S$, mansoni. Publicações Avulsas do Instituto Aggeu Magalhães 3:55-62, 1954.

3. Bernardini OJ, Machado MM. Esquistossomose mansoni em Santa Catarina: isolamento do Scbistosoma mansoni do primeiro foco de transmissão ativa em São Francisco do Sul. Nota Prévia. Arquivos Catarinenses de Medicina 10:213, 1981.

4. Carvalho OS, Guimarães CT, Massara CL, Bonesio JER. Situação atual da esquistossomose mansoni no lago da Pampulha, Belo Horizonte, MG, Brasil. Revista de Saúde Pública 19: 270-277, 1985.

5. Carvalho OS, Massara CL, Silveira Neto HV, Alvarenga AG, Vidigal THDA, Guerra HL, Santos MAA, Katz N. Schistosomiasis mansoni in the region of the triângulo mineiro - State of Minas Gerais, Brazil. Memórias do Instituto Oswaldo Cruz 89:509-512, 1994

6. Carvalho OS, Souza CP, Katz N. Primeiro encontro de Biomphalaria tenagophila (d'Orbigny, 1835) naturalmente infectada com Schistosoma mansoni, em Itajubá, sul do estado de Minas Gerais, Brasil. Revista de Saúde Pública 19:88-91, 1985.

7. Correa RR, Piza JT, Ramos A, Camargo LV. Planorbideos do Estado de São Paulo: sua relação com a esquistossomose. Arquivos de Higiene e Saúde Pública 27:139-159, 1962.

8. Espíndola KS, Hofmann PRP, Machado MM. Preliminary survey of the planorbidic fauna in Santa Catarina Island, Santa Catarina, Brazil. Memórias do Instituto Oswaldo Cruz 85:375, 1990.

9. Frandsen F. Discussion of the relationships between Schistosoma and their intermediate hosts, assessment of the degree of host-parasite compatibility and evaluation of schistosome taxonomy. Zeitschrift für Parasitenkunde 58:275$296,1979$.

10. Freitas JR, Santos MBL, Lima LZ, Rocha LA, Souza CP. Distribuição dos moluscos hospedeiros intermediários do Schistosoma mansoni na bacia da Pampulha, Belo Horizonte, MG, Atualização de dados. In: Anais da $43^{a}$ Reunião Anual da SBPC, Ciência e Cultura 43(supl):641-642, 1991.

11. Katz N, Chaves A, Pellegrino J.A simple device for quantitative stool thick smear technique in schistosomiasis mansoni. Revista do Instituto de Medicina Tropical de São Paulo 14:397-402, 1972.

12. Melo AL, Pereira LH. On the finding of Biomphalaria tenagophila naturally infected with Schistosoma mansoni in the state of Minas Gerais, Brazil. Revista do Instituto de Medicina Tropical de São Paulo 27:99-101, 1985.

13. Paraense WL. Fauna planorbídica do Brasil. In: Lacaz CS, Baruzzi GR, Siqueira JRW (eds) 
Sonza CP, Jannotti-Passos LK, Vieira IBF, Sampaio IBM. Schistosoma mansoni: nineis de infecciosidade para os moluscos bospedeiros intermediários do Brasil. Relista da Sociedade Brasileira de Medicina Tropical 29:1116, jan-fev? 1996.

Introdução à geografia médica do Brasil, Edgard Blucher e Editora Universidade de São Paulo p. 13-20, 1972.

14. Paraense WL. A survey of planorbid molluses in the Amazonian region of Brazil. Memórias do Instituto Oswaldo Cruz 78: 343-361, 1983.

15. Paraense WL. Distribuição dos caramujos no Brasil - In: Reis FA, Faria I, Katz N (eds) Modernos conhecimentos sobre esquistossomose mansônica, Biblioteca Academia Mineira de Medicina 14:117$128,1986$.

16. Paraense WL, Alencar AJT, Correa LR. Distribuição dos planorbídeos e prevalência da xistosomose mansoni no Estado do Espírito Santo. Memórias do Instituto Oswaldo Cruz 78:373-384, 1983.

17. Paraense WL, Correa LR. Sobre a ocorrência de duas raças biológicas de Schistosoma mansoni no Brasil. In: Resumos da XV Reunião Anual da Socicdade Brasileira para o Progresso da Ciência, Resumos, Campinas, SP, 1963.

18. Paraense WL, Correa LR. Differencial susceptibility of Biomphalatia tenagopbila populations to infection with a strain of Schistosoma mansoni. Journal of Parasitology 64:822-826, 1978.

19. Paraense WL, Correa LR. Observations of two biological races of Scbistosoma mansoni. Memórias do Instituto Oswaldo Cruz 76:287-291, 1981.

20. Paraense WL, Correa LR. Probable extension of schistosomiasis mansoni to southermost Brazil. Memórias do Instituto Oswaldo Cruz 82:577, 1987.

21. Paraense WL, Correa LR. A potencial vector of Schistosoma mansoni in Uruguay. Memórias do Instituto Oswaldo Cruz 84:281-288, 1989.

22. Paulini E. Da aplicação de moluscicidas: VI Nomograma para o cálculo de moluscicidas. Revista Brasileira de Malariologia e Doenças Tropicais 20:225-229,1968.
23. Pellegrino J, Katz N. Experimental chemotherapy of schistosomiasis mansoni. In: Dawes B (ed) Advances in Parasitology,Academic Press, London 6:233-290, 1968.

24. Snedecor G W, Cochran WG. Statistical methods, 6th edition University Press Ames, 1968.

25. Silva RE. Estudo malacológico da bacia hidrográfica do lago Soledade (Ouro Branco, Minas Gerais): testes de suscetibilidade com os transmissores do Scbistosoma mansoni. Caracterização de trematódeos emergentes de moluscos. Tese de Mestrado, Universidade Federal de Minas Gerais, Belo Horizonte, MG, 1992.

26. Souza CP, Dias EP, Azevedo MLL, Paulini E. Observações sobre alguns fatores que influem na manutenção do Scbistosoma manısoni em laboratório. Revista Brasileira de Pesquisas Médicas e Biológicas 12:411-419, 1979.

27. Souza CP, Jannotti- Passos LK, Freitas JR. Degree of host parasite compatibility between Scbistosoma mansoni and their intermediate molluscan hosts in Brazil. Memórias do Instituto Oswaldo Cruz, 90:5-10, 1995.

28. Souza CP, Lima LC. Moluscos de interesse parasitológico do Brasil. Série Esquistossomose $n^{\circ}$ 1, FIOCRUZ -CPqRR, Belo Horizonte, MG, 1990.

29. Teles HMS, Leite RPA, Rodrigues FL. Moluscos límnicos de uma área do Alto Araguaia (Brasil). Revista de Saúde Pública 25:179-183, 1991.

30. Teles HMS, Pereira PAC, Richinitti LMZ. Distribuição de Biomphalaria (Gastropoda; Planorbidae) nos estados do Rio Grande do Sul c Santa Catarina, Brasil. Revista de Saúde Pública 25:350-352, 1991.

31. Valadão R, Milward De Andrade R. Interações de planorbideos vetores de esquistossomose mansoni e o problema da expansão da endemia na região amazônica. Revista de Saúde Pública 25:353-358, 1991. 